\title{
Quantitative estimation of the corrosion rate of metallic materials using confocal laser scanning microscopy
}

\author{
V. A. Danilov ${ }^{\dagger}$, D. L. Merson \\ †VA.Danilov@yahoo.com \\ Institute of Advanced Technologies, Togliatti State University, Togliatti, 445020, Russia
}

This study represents an alternative method for quantitative estimation of the corrosion rate of various metallic materials, in particular magnesium and its alloys, using confocal laser scanning microscopy (CLSM). This method makes it possible to obtain not only optical images of the specimen surface, but also accurate 3D height maps which allow a quantitative assessment of many characteristics such as surface profile, damage locality, number and maximum depth of corrosion pits, volume of metal loss and corrosion products. Specific methodological aspects and possibilities of using the CLSM method for the analysis of corrosion damage have been described. The proposed method is based on obtaining 3D images of the surface of specimens after corrosion tests with their subsequent processing and analysis, with the use of original software computer procedures for digital image processing and topographic data arrays. A technique for recording and subsequent analysis of specimens after corrosion tests using CLSM has been developed and tested. The optimal magnification, lens type, scanning step and noise filtering have been determined. The procedure prescribes mounting the test metallic sample into a non-corrosive material to create a stable reference plane surface. The dissolution of the metal at the specimen-mount interface is found to be notably more intensive than in the specimen interior when the conventional metallographic resins are used as the mount. This fact deteriorates the objectivity of the results of corrosion rate measurements. Polyethylene is proposed as the mounting material to minimize this effect. The geometry and the minimum size of the metal sample, providing a reduction in labor contribution for scanning and data processing without loss of accuracy and reliability of the data are established. The values of the uniform corrosion rate of commercially pure magnesium in Ringer's solution using the gravimetric method and the CLSM method showed satisfactory repeatability of measurements.

Keywords: confocal laser scanning microscopy, metal corrosion, corrosion rate, corrosion test technique, quantitative eastimation of local corrosion rate.

УДК: 620.193 .2

\section{Количественная оценка скорости коррозии металлических материалов с помощью конфокальной лазерной сканирующей микроскопии}

\author{
Данилов В. А. ${ }^{\dagger}$, Мерсон Д. Л. \\ Научно-исследовательский институт прогрессивных технологий, Тольяттинский государственный университет, \\ Тольятти, 445020, Россия
}

Работа посвящена разработке альтернативной методики количественной оценки скорости коррозии различных металлических материалов, в частности магния и его сплавов, при помощи конфокальной лазерной сканирующей микроскопии (КЛСМ). Данный метод позволяет не только получать оптические изображения поверхности образца, но и точные 3D карты высот, позволяющие количественно определять такие характеристики, как профиль поверхности, локальность повреждения, количество и максимальная глубина коррозионных язв, объем потерянного металла и продуктов коррозии. Описаны методологические особенности и возможности применения 
метода КЛСМ для анализа коррозионных повреждений. В основе предлагаемой методики лежит получение 3D-снимков поверхности образцов после коррозионных испытаний с последующей их обработкой и анализом, в том числе с использованием оригинальных программных компьютерных процедур цифровой обработки изображений и массивов топографических данных. Разработана и апробирована методика съемки и последующего анализа образцов после коррозионных испытаний при помощи КЛСМ. Установлены оптимальные увеличение, тип объектива, шаг сканирования и способ фильтрации получаемых изображений от шумов. Для создания стабильно существующей во времени плоскости отсчета методика предписывает заливку испытуемого образца в некорродирующий материал. Согласно проведенным исследованиям при использовании стандартных смол, применяемых в металлографии, наблюдается более интенсивное растворение испытуемого металла на границе образец-шлиф, чем в теле самого образца, что приводит к снижению объективности результатов измерения скорости коррозии. В качестве материала заливки, минимизирующего этот эффект, предложен полиэтилен. Установлены геометрия и минимальный размер образца, обеспечивающие снижение трудозатрат на съёмку и обработку данных без потери точности и достоверности данных. Измеренные значения скорости равномерной коррозии технически чистого магния в растворе Рингера с помощью гравиметрического метода и метода КЛСМ показали удовлетворительную сходимость.

Ключевые слова: конфокальная лазерная сканирующая микроскопия, коррозия металлов, скорость коррозии, методика коррозионных испытаний, количественная оценка скорости локальной коррозии.

\section{1. Введение}

Процессы коррозии являются одной из основных причин выхода из строя различных машин, аппаратов и металлоконструкций, поэтому их необходимо обнаруживать как можно на более ранних стадиях. Для этого требуются надежные методики, позволяющие количественно оценивать коррозионные повреждения с целью получения точного прогноза последующего развития процессов коррозии. Установление причин коррозионного разрушения металла изделия является важной задачей, т. к. позволяет корректно выбрать метод защиты. Поэтому целью коррозионных испытаний является не только определение коррозионной стойкости конкретного металла в определенных условиях, но и изучение самого механизма коррозии. Работы в этом направлении ведут многие исследователи [1-4]. Среди методов количественной оценки скорости коррозии, наиболее распространенным является гравиметрический (весовой) метод [5,6], заключающийся в измерении убыли массы образца в процессе выдержки в агрессивной среде в течение известного интервала времени. Другой, объемный метод оценки скорости коррозии, основан на измерении количества выделившегося водорода, прямо пропорционального потере веса испытуемого материала [7-9]. В последние годы активно применяются непрерывные методы контроля скорости коррозии [10]: методы поляризационного и электрического сопротивления. В основу метода поляризационного сопротивления положена наблюдаемая во многих случаях вблизи потенциала коррозии линейная зависимость между величиной поляризующего тока и вызываемого им изменения потенциала [11], однако он может быть применен только в ограниченных условиях. Метод электрического сопротивления в отличие от предыдущего может применяться в любых средах, как электропроводных, так и неэлектропроводных.

Основным недостатком существующих на сегодня методов по оценке коррозионной повреждаемости материалов является невозможность определения количест- венных показателей, характеризующих рельеф коррозионных повреждений (глубина и поперечные размеры питтинга, глубина проникновения межкристаллитной коррозии и др.), и, как следствие, невозможность идентификации типа коррозионного повреждения (язвенная, питтинговая и др.). Таким образом, существует острая необходимость в более точных и эффективных методах исследования и оценки коррозионной повреждаемости, способных не только рассчитать общую коррозию, но и количественно описывать разные типы коррозии.

Для решения данной задачи представляется перспективным использование конфокального лазерного сканирующего микроскопа (КЛСМ), уже хорошо зарекомендовавшего себя, как прецизионное оборудование для решения различных металловедческих задач [12-15], в том числе в работах нашего коллектива [16-17]. Современные приборы, использующие метод КЛСМ, позволяют получать трехмерные изображения поверхности в широком диапазоне сканирования с высокой разрешающей способностью. Метод КЛСМ позволяет с высочайшей точностью определять объем прокорродировавшего металла даже при очень малых его значениях, т.е. он является принципиально более чувствительным, чем гравиметрический метод, благодаря чему позволяет значительно снизить необходимое время экспозиции контрольных образцов в коррозионной среде и трудоемкость коррозионных испытаний в целом.

Однако для внедрения метода КЛСМ в практику коррозионных испытаний требуется разработать и апробировать методику, как в части получения изображений, так и в части проведения самих коррозионных испытаний, поскольку от параметров съемки и подготовки образцов к испытаниям могут существенно зависеть искомые величины (площадь и объем коррозионных повреждений коррозии, глубина и т.п.). В связи с этим требовалось: подобрать объектив и увеличение съемки, шаг сканирования вдоль оси Z, выбрать фильтр для устранения оптических шумов, подобрать материал шлифа, определить оптимальную геометрию и размеры образца. 


\section{2. Объекты и методы исследования}

Для проведения экспериментов использовали заготовки из монокристалла (99.8 масс.\%) и технически чистого магния (99.8 масс.\%). Вырезку монокристалла осуществляли с помощью прецизионного отрезного станка Buehler IsoMet, a технически чистого магния - c помощью электроискрового проволочно-вырезного станка Sodick AG400L LN2W. Средний размер зерна технически чистого магния после прокатки и отжига $\left(300^{\circ} \mathrm{C}\right.$, 30 мин.) составлял 27 мкм. Образцы запрессовывали в различные виды заливки с использованием станка Buehler SIMPLIMET 1000. Чтобы исключить влияние микроструктуры и неоднородности химического состава на коррозию для экспериментов был выбран монокристалл магния, который для чистоты эксперимента во всех случаях был ориентирован к рабочей поверхности модельной плоскостью (0001). Определение элементного состава заливок проводили после их полимеризации с помощью энергодисперсионного рентгенофлуоресцентного спектрометра EDX-8000.

Шлиф с образцом вручную шлифовался до получения общей плоской поверхности металла с застывшей заливкой на шлифовально-полировальном станке Struers LaboPol-25 наждачной бумагой зернистостью 600, 2500 и 4000 под проточной водой до шероховатости $R_{a} 0.3$ мкм. После шлифования образцы промывали в изопропиловом спирте, обезжиривали в течение 3-5 минут в ультразвуковой ванне и быстро высушивали струёй воздуха.

Готовые шлифы устанавливались в испытательный стенд для коррозионных испытаний, в состав которого входили: стеклянная камера с коррозионным раствором объёмом 3 литра; циркуляционный термостат LOIP LT-111P, поддерживающий заданную температуру; насос перистальтический LOIP LS-301, осуществляющий перемешивание коррозионной среды; электронный $\mathrm{pH}$-метр/ионометр для контроля и фиксации значений $\mathrm{pH}$ и $T$ раствора с записью на персональный компьютер. Так как магний является перспективным материалом для создания медицинских биорезорбируемых имплантатов для человеческого организма [18], в качестве коррозионной среды использовался раствор Рингера (фирмы Солофарм) следующего состава: 8.6 г/л NaCl, 0.3 г/л $\mathrm{KCl}, 0.25$ г/л $\mathrm{CaCl}_{2}$. В ходе коррозионных испытаний поддерживались температура жидкости $37.0 \pm 0.1^{\circ} \mathrm{C}$ и уровень $\mathrm{pH} 7.4 \pm 0.5$. После испытаний продукты коррозии удаляли промывкой в растворе, содержащем: 200 г/л $\mathrm{CrO}_{3}, 10$ г/л $\mathrm{AgNO}_{3}$ и дистиллированную воду (ГОСТ Р 9.907-2007). Образцы взвешивали до и после коррозионных испытаний с использованием аналитических весов с точностью 0.0001 г.

Топологию поверхности до и после коррозионных испытаний исследовали с помощью конфокального лазерного сканирующего микроскопа Olympus LEXT OLS 4000. Данный прибор способен работать в широком диапазоне сканирования вдоль оси $\mathrm{Z}$ с разрешающей способностью до 110 нм в плоскости XY и до 10 нм вдоль оси Z, что позволяет производить качественный и количественный анализ прокорродировавшей поверхности с высокой точностью.

\section{3. Результаты и обсуждение}

\section{1. Методика определения коррозионной повреждаемости металлических материалов}

\subsection{1. Оптимальные параметры съёмки}

Поскольку исследуемая поверхность после испытаний имеет достаточно сложный с большими перепадами рельеф, для получения ее качественной картины требовалось отработать оптимальные режимы построения изображений с помощью КЛСМ, а именно: подобрать объектив, увеличение, шаг сканирования и фильтр для устранения оптических шумов. Оказалось, что для оценки коррозионных повреждений наиболее эффективны те же самые параметры, что и для исследования морфологии изломов [19], а именно: (1) объектив MPLAPON20XLEXT обеспечивает высокую разрешающую способность при увеличении 400× и безопасное расстояние объектива до исследуемой поверхности; (2) фильтр «pre-measurement» обеспечивает полное удаление шумов и минимальное искажение реальной поверхности; (3) шаг сканирования вдоль оси Z, равный 0.8 мкм, обеспечивает достаточное разрешение при минимальном времени съёмки.

\subsection{2. Методика вычисления объёма потерянного металла}

На Рис. 1 изображены панорамные снимки образцов: до (Рис. 1a) и после коррозионных испытаний (Рис. 1b), и после снятия продуктов коррозии (Рис. 1c). Подготовленная к испытаниям поверхность заливки с образцом представляют собой базовую плоскость («нулевой уровень»), поэтому до начала испытаний ее профилограмма имеет вид прямой горизонтальной линии (Рис. 1a). У образца с продуктами коррозии (ПК) (Рис. $1 \mathrm{~b})$ имеются отклонения от базовой плоскости как вверх, так и вниз, т.е. при коррозионных процессах происходит как растворение металла, так и оседание ПК. На профилограммах Рис. 1 область, относящаяся непосредственно к испытуемому образцу, ограничена вертикальными линиями.

Как и следовало ожидать, после удаления ПК наблюдающиеся на поверхности образца отклонения от базовой плоскости происходят только в глубину (Рис. 1c). Площадь этих отклонений, находящаяся под «нулевым уровнем», выделена на Рис. 1 красным цветом. Интегрируя эту площадь по всему образцу, можно оценить полный объём прокорродированного металла и с учетом времени экспозиции образца в коррозионной среде рассчитать скорость коррозии.

\subsection{3. Выбор заливки образиа}

С целью проверки возможного негативного влияния заливки на коррозионные процессы испытуемых образцов, были исследованы 5 типов заливок: формальдегидная смола с графитовым наполнителем - PFD (peжим: $T=180^{\circ} \mathrm{C}, p=300 \mathrm{bar}$ ); формальдегидная смола $-\mathrm{PF}$ 
(режим: $T=180^{\circ} \mathrm{C}, p=300$ бар); акриловая смола - DC (режим: $T=25^{\circ} \mathrm{C}, t=24$ ч); эпоксидная смола - EF (режим: $T=25^{\circ} \mathrm{C}, t=24$ ч) и полиэтилен $-\mathrm{LDPE}$ (режим: $T=150^{\circ} \mathrm{C}, p=270$ бар).

На Рис. 2 представлены панорамные изображения и профили поверхности образцов монокристалла магния, запрессованных в различные виды заливки, после коррозионных испытаний в растворе Рингера в течение 2 (Рис. 2a) и 96 часов (Рис. 2b-e). Съёмка производилась при параметрах, указанных в п. 3.1.1. При увеличении 400× для съёмки всей поверхности образцов необходимо построение панорамного изображения из 16 кадров $(4 \times 4)$ с площадью каждого из них $640 \times 640$ мкм. Анализ влияния типа заливки на коррозию на границе образецзаливка показал следующее.

Заливка PFD (Рис. 2а) показала худший результат, т. к. сразу после погружения образца в коррозионную среду началось активное выделение водорода, свидетельствующее о высокой скорости коррозии. Уже после 2 часов глубина проникновения коррозии составила 260 мкм. Образцы в заливках PF, DC и EF (Рис. 2 b-d) имели равномерный тип коррозии, проникающий на глубину около 30 мкм. Однако по краям образцов, граничащих с заливкой, наблюдалась существенно более интенсивная коррозия с глубиной проникновения около 60 мкм (на Рис. 2 отмечены красными кругами),
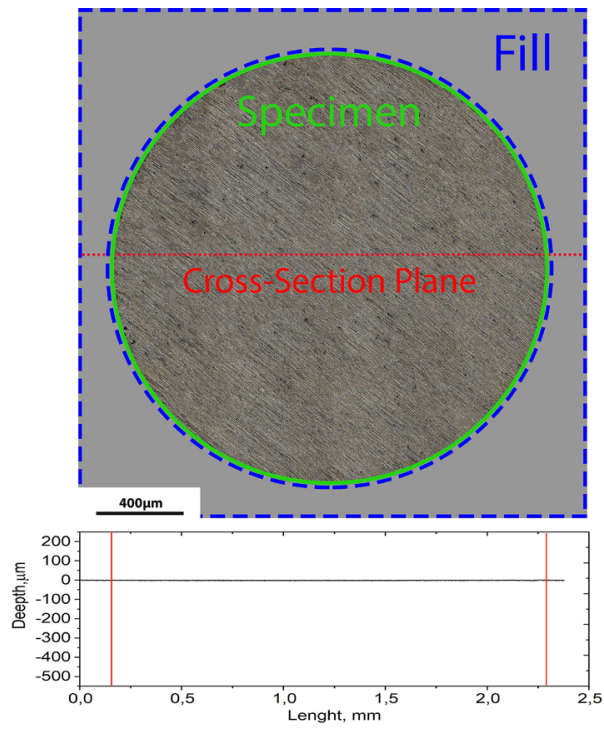

a
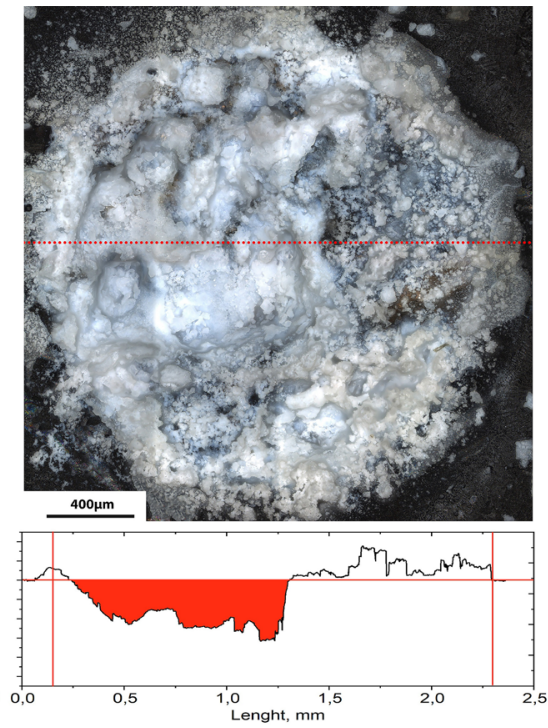

b
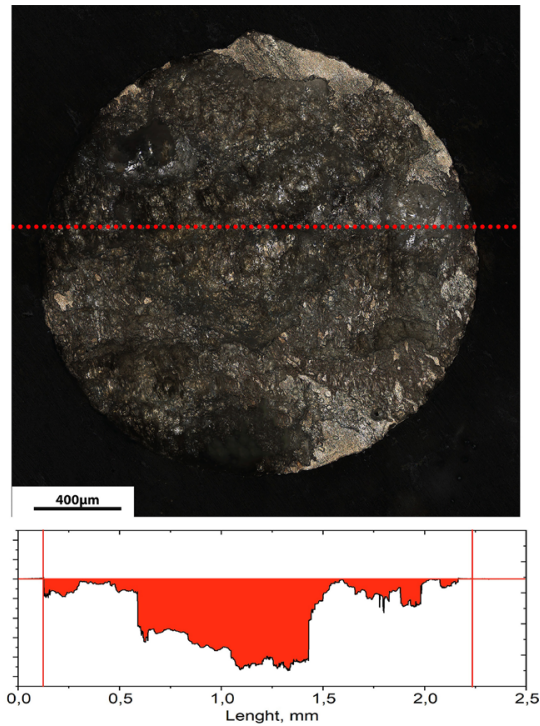

c

Pис. 1. (Color online) Панорамные изображения поверхности образцов, полученные с помощью КЛСМ, и методика определения объёма металла, потерянного в результате коррозии: до коррозионных испытаний (a), после выдержки в растворе Рингера (b), после удаления продуктов коррозии (c).

Fig. 1. (Color online) Panoramic images of sample surface obtained using CLSM and methods for determining the volume of metal lost as a result of corrosion: before corrosion tests (a), after holding in Ringer's solution (b), after removal of corrosion products (c).
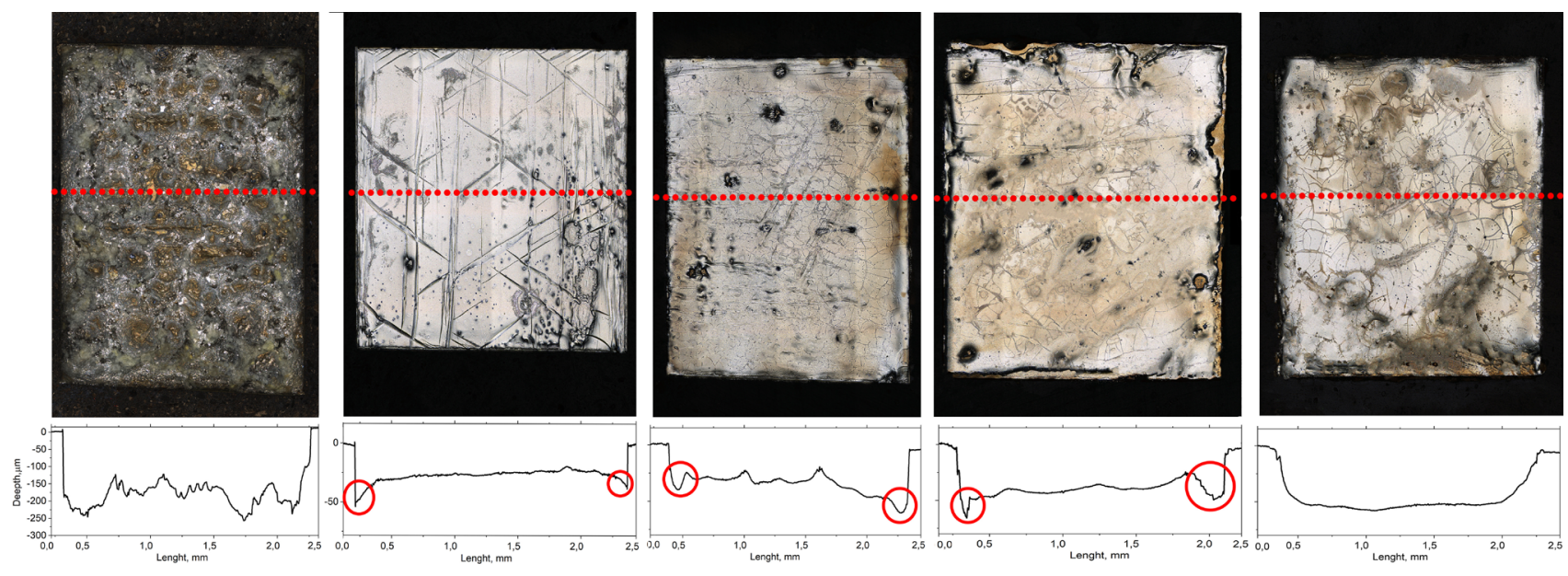

a

$\mathrm{b}$

d

$\mathrm{e}$

Pис. 2. (Color online) Панорамные изображения образцов магния из монокристалла в разных заливках; в нижней строке показаны соответствующие профили, полученные по штриховым линиям после удаления продуктов коррозии: PFD (a), PF (b), DC (c), EF (d), $\operatorname{LDPE}(\mathrm{e})$.

Fig. 2. (Color online) Panoramic images of magnesium samples from a single crystal in different fills; the corresponding profiles obtained on the dashed lines after removal of corrosion products are shown: PFD (a), PF (b), DC (c), EF (d), LDPE (e). 
что, вероятно, связано с наличием «вредных» примесей в составе заливок. Кроме того, на Рис. 2 b отчётливо видны линии скольжения, образование которых, по-видимому, связано с инициацией источников дислокаций под действием высокой температуры $\left(180^{\circ} \mathrm{C}\right)$ и большого давления (300 бар) при подготовке образца. Элементный анализ показал присутствие в составе шлифа $\mathrm{Fe}, \mathrm{Si}, \mathrm{S}$, $\mathrm{Al}$ и $\mathrm{Cr}$, с некоторыми из которых на границе образецзаливка, по-видимому, образуются гальванические пары, способствующие увеличению скорости коррозии. Наилучший результат показала заливка LDPE (Рис. 2е), при использовании которой, в отличие от всех других исследованных типов заливок, наблюдался равномерный тип коррозии по всему образцу с отсутствием «провалов» на границе образца-заливки. Вероятно, в составе LDPE «вредные» примеси отсутствуют. Это подтверждается и литературными данными [20], согласно которым полиэтилен состоит только из углерода и водорода и фактически не имеет примесей. Дополнительным плюсом полиэтилена является отсутствие разбухания и абсолютная водонепроницаемость [21], что позволяет при использовании массового метода минимизировать погрешности, связанные с изменением веса шлифа. Таким образом, использование LDPE в качестве заливки является предпочтительным в методике оценки коррозионной повреждаемости металлических образцов с помощью метода КЛСМ.

\subsection{4. Влияние геометрии образиов}

С целью проверки влияния геометрии испытуемых образцов на результаты оценки скорости коррозии были изготовлены круглые (диаметр 2 мм) и квадратные (сторона 2 мм) образцы, которые запрессовывали в заливку LDPE (оранжевая область на Рис. 3) и выдерживали в растворе Рингера 96 часов. После удаления продуктов коррозии выполняли панорамную съёмку с выше описанными оптимальными параметрами. На Рис. 3 приведены изображения карты высот поверхности образцов монокристалла магния, ориентированных плоскостью (0001) к рабочей поверхности образца: в

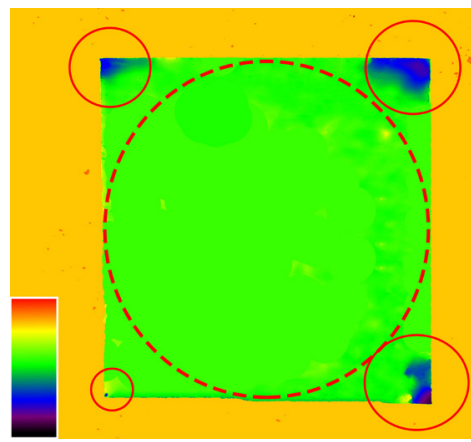

Pис. 3. (Color online) Карты высот образцов монокристалла магния разной геометрии после коррозионных испытаний в растворе Рингера в течение 96 часов и снятия продуктов коррозии.

Fig. 3. (Color online) Height maps of magnesium single crystal samples of different geometries after corrosion tests in Ringer's solution for 96 hours and removal of corrosion products. виде круга (пунктирная ограничивающая линия) и квадрата (зелёная область).

На обоих типах образцов наблюдается преимущественно равномерный тип коррозии. Однако в углах образцов квадратного сечения имеются участки с локальным коррозионным повреждением, которые выделены красными кругами. Глубина коррозионного повреждения в углах достигает 50 мкм, при глубине общей коррозии в 30 мкм. На круглых образцах вся поверхность образца имеет равномерное коррозионное повреждение без участков локальной коррозии.

Следовательно, геометрия образца играет немаловажную роль, а использование образцов круглого сечения позволяет повысить точность и достоверность получаемых данных коррозионных испытаний.

\subsection{5. Влияние размера образиа}

Поскольку время сканирования напрямую определяется размерами образца, требовалось подобрать его минимально возможные размеры без ущерба точности измеряемых величин. Образцы технически чистого магния круглого сечения с диаметрами от 0.5 до 5 мм запрессовывали в заливку LDPE, шлифовали, помещали в коррозионную среду на 24 часа, удаляли продукты коррозии и проводили панорамную съёмку с помощью КЛСМ при ранее подобранных параметрах. Размер панорамы определяется диаметром образца, поэтому в качестве меры сравнения служило отношение объёма потерянного металла к площади образца (V/S).

На Рис. 4. изображён график, показывающий зависимости времени, затраченного на съёмку образцов, и параметра V/S от диаметра образца. Видно, что с увеличением диаметра, время, затраченное на съёмку, непрерывно увеличивается, а параметр V/S сначала резко снижается, но после достижения 2 мм стабилизируется на одном уровне. Кроме того, начиная с диаметра 2 мм и разброс данных существенно снижается. Таким образом, для методики оценки параметров скорости коррозии с помощью метода КЛСМ оптимальным размером круглых образцов является диаметр 2 мм.

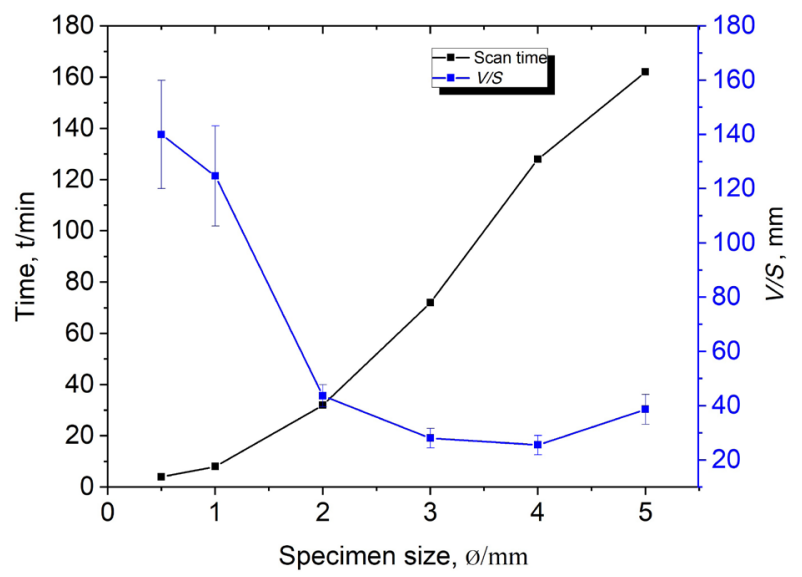

Pис. 4. (Color online) Зависимости времени съёмки и отношения объёма потерянного металла к площади образца от диаметра.

Fig. 4. (Color online) Dependences of the scanning time and the ratioes of the volume of the metal loss to the sample area to the diameter. 


\section{2. Сравнение скорости коррозии, определенной методом КЛСМ и весовым методом}

Для апробации методики был проведён эксперимент по сравнению данных скоростей коррозии, определенных по разработанной методике метода КЛСМ и методике стандартного гравиметрического метода (ГОСТ 9.913-90). Каждый из 5 образцов технически чистого магния диаметром 5 мм заливали в полиэтилен, подготавливали поверхность с шероховатостью 0.3 мкм, взвешивали шлифы с точностью до 0.0001 г. Увеличение диаметра образца от оптимального размера (2 мм) до 5 мм продиктовано требованием обеспечения достаточной чувствительности весового метода. После коррозионных испытаний в растворе Рингера в течение 48 часов образцы извлекали, удаляли продукты коррозии, шлифы повторно взвешивали и вычисляли скорость коррозии $V_{\text {corr }}$ Определение $V_{\text {corr }}$ по данным КЛСМ проводили исходя из объёма потерянного металла, вычисленного по данным панорамной съёмки образцов.

Измеренная по 5 образцам средняя $V_{\text {corr }}$ составила 24 и 26 мм/год, соответственно по данным весового метода и метода КЛСМ. Анализ поверхности по данным КЛСМ показал, что морфология полученных образцов (Рис. 5) характеризуется наличием как общей, так и локальной (язвенной) коррозии, с преобладанием последней. Вероятнее всего, коррозия протекает по «благоприятно» ориентированным зернам в образце, в то время как другие остаются практически нетронутыми. Максимальная глубина проникновения коррозии составила 530 мкм, что соответствует скорости локальной коррозии 97 мм/год. Погрешность измерений между образцами

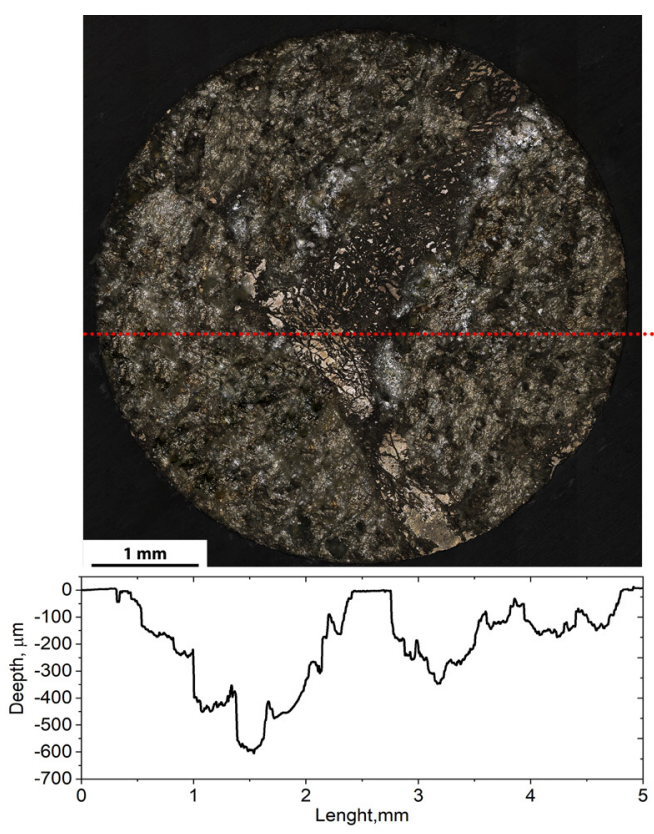

Pис. 5. (Color online) Морфология с профилограммой коррозионного повреждения технически чистого магния после выдержки в растворе Рингера в течение 48 часов.

Fig. 5. (Color online) Morphology with a profilogram of corrosion damage of commercially pure magnesium after holding in Ringer's solution for 48 hours. по методу КЛСМ составила около $10 \%$, а разница значений между двумя методами 7\%. Таким образом, оба метода показывают практически идентичный результат значений скорости коррозии. Разница между значениями скоростей коррозии, полученными двумя методами, может быть обусловлена тем, что объем потерянного металла вычисляется с помощью КЛСМ непосредственно в испытуемом образце, а измерение веса до и после испытаний - в шлифе.

\section{4. Выводы}

1. Впервые разработана методика определения коррозионной повреждаемости металлических материалов, основанная на методе КЛСМ. Созданная методика содержит оптимальные параметры съёмки и обработки, а также методические аспекты пробоподготовки и позволяет получать на основе высококачественных трёхмерных изображений прокорродировавшей поверхности объективные количественные данные о характере коррозии, объеме потерянного металла и скоростях равномерной и локальной коррозии.

2. Применение полиэтилена в качестве заливки позволяет исключить влияние «вредных» примесей на скорость коррозии, а круглое сечение - нивелировать влияние на нее геометрического фактора. Диаметр испытуемого образца величиной 2 мм является оптимальным, т.к. позволяет минимизировать трудоемкость процесса сканирования без потери точности получения результатов.

3. Сравнение результатов оценки скорости равномерной коррозии с помощью разработанной методики и стандартного массового метода показало удовлетворительную сходимость значений, но при этом метод КЛСМ дополнительно дает полную информацию о морфологии коррозионных повреждений и позволяет количественно оценивать параметры локальной коррозии.

Благодарности / Aknowledgements. Исследование выполнено при финансовой поддержке РФФИ в рамках научного проекта № 19-38-90090. / The reported study was funded by RFBR, project number 19-38-90090.

\section{Литература/References}

1. C. Andrade, C. Alonso. Construction and building materials. 15 (2-3), 141 (2001). Crossref

2. A. Neville, T. Hodgkiess, H.Xu. Wear. 233-235, 523 (1999). Crossref

3. L. G. Bland et al. Corrosion. 71 (2), 128 (2015). Crossref

4. F. Rosalbino, G. Scavino. Electrochimica acta. 111, 656 (2013). Crossref

5. E. Stupnišek-Lisac, A. Gazivoda, M. Madžarac. Electrochimica acta. 47 (26), 4189 (2002). Crossref

6. P. Kwolek. RSC Advances. 10 (44), 26078 (2020). Crossref

7. S. Thomas, N. V. Medhekar, G. S. Frankel, N. Birbilis. Curr. Open. Solid. State. Mater. Sci. 19 (2), 85 (2015). Crossref

8. T. Haruna, D. Nishiwaki, M. Nishikawa. Materials Science Forum. 794-796, 107 (2014). Crossref 
9. R. Solmaz, G. Kardas. Energy Conversion and Management. 48 (2), 583 (2007). Crossref

10. F. Bentiss, M. Lagrenee, M. Traisnel, J.C. Hornez. Corrosion Science. 41 (4), 783 (1999). Crossref

11. R.G. Kelly, J.R. Scully, D. Shoesmith, R.G. Buchheit. Electrochemical Techniques in Corrosion Science and Engineering. CRC Press (2002). Crossref

12. U. Trdan, M. Skarbab, J. A. Porro, J. L. Ocaña, J. Gruma. Surf. Coat. Tech. 342, 1 (2018). Crossref

13. L. C. de Paula, S. Tokita, K. Kadoi, H. Inoue, E. J. Zoqui. Solid State Phenomena. 285, 277 (2019). Crossref

14. U. Wendt, K. Stiebe-Lange, M. Smid, K.D. Tönnies. Microsc. Microanal. 9 (S03), 370 (2003). Crossref

15. M. Stratigaki, C. Baumann, L.C. A. van Breemen, J. P. A. Heuts, R. P. Sijbesma, R. Göstl. Polymer Chemistry. 11 (2), 358 (2020). $\underline{\text { Crossref }}$

16. E. Merson, V. Danilov, D. Merson, A. Vinogradov. Eng.
Fract. Mech. 183, 147 (2017). Crossref

17. E.D. Merson, V.A. Danilov, M.L. Linderov, P. N. Myagkikh, D.L. Merson, A. Vinogradov. Procedia Structural Integrity. 13, 2152 (2018). Crossref

18. D.L. Merson, A.I. Brilevsky, P.N. Myagkikh, M. V. Markushev, A. Vinogradov. Lett. Mater. 10 (2), 217 (2020). Crossref

19. E. D. Merson, V. A. Danilov, D. L. Merson. Vector Science of Togliatti State University. 4 (34), 68 (2015). (in Russian) [Е.Д. Мерсон, В.А. Данилов, Д. Л. Мерсон. Вектор науки Тольяттинского государственного университет. 4 (34), 68 (2015).] Crossref

20. T. Okamura, S. Kobayashi, K. Müllen. Encyclopedia of Polymeric Nanomaterials. Springer, Berlin, Heidelberg (2014). Crossref

21. X. Zhong, X. Zhao, Y. Qian, Y.Zou. Insight - Material Science. 1 (1), 1 (2017). Crossref 